\title{
Características de um Grupo de Hipertensos em uma USF da Cidade Cariri do Tocantins
}

\section{Characteristics of a Group of Hypertensive Patients at a USF in Cariri do Tocantins}

Sandra Nara Marroni ${ }^{\text {, }}$, Jaileide Cecilia de Macedo ${ }^{2}$, Luana Menezes Silva ${ }^{3}$,

Márcia Andréa Marroni ${ }^{4}$

RESUMO

Introdução: A Hipertensão Arterial é um grande problema de saúde pública mundial e no Brasil, caracterizada por níveis elevados de pressão arterial, agravando-se na presença de fatores de risco como obesidade, dislipidemias e sedentarismo. Objetivo: Caracterizar um grupo de hipertensos em uma Unidade de Saúde da Família - USFdo município de Cariri do Tocantins quanto aos dados biossociais, tratamento, hábitos e estilo de vida e alguns riscos para desenvolvimento de doença cardiovascular. Material e métodos: Estudo exploratório, descritivo transversal, de natureza quantitativa, realizado com 162 pacientes no período de agosto a outubro de 2018. Foram incluídos pacientes hipertensos, maiores de 18 anos caracterizados por entrevista semiestruturada. $O$ questionário foi aplicado nas consultas e domicílios. A tabulação dos dados foi feita em planilha Excel 2016, e apresentadas em forma de tabelas com frequências absolutas e relativas. Resultados: o maior contingente de pesquisados foram mulheres (65\%) e idosos, entre 59 e acima de 65 anos (73\%), com baixo índice de escolaridade $(70 \%)$. A diabetes $(27 \%)$ e o sedentarismo $(58 \%)$ foram os fatores de risco mais predominantes. A população feminina apresentou maior índice de obesidade abdominal (48\%). Conclusão: O perfil dos hipertensos desta USF integra maior parte pacientes do sexo feminino, idade avançada e apresentam fatores de risco para desenvolvimento de doenças cardiovasculares que merecem maior assistência. As informações adquiridas podem contribuir para a equipe de saúde conhecer melhor sua população e desenvolver estratégias de intervenções acerca do tratamento e acompanhamento para controlar a doença e melhorar a qualidade de vida dos mesmos.

Descritores: Hipertensão. Doenças cardiovasculares. Fatores de risco.

\section{ABSTRACT}

Introduction: Hypertension is a major public health problem in the world and in Brazil, characterized by high blood pressure levels, worsening in the presence of some risk factors such as obesity, dyslipidemia and sedentary lifestyle. Objective: To characterize a group of hypertensive patients in a Family Health Unit - USF in the city of Cariri do Tocantins regarding biosocial data, treatment, habits and lifestyle and some risks for the development of cardiovascular disease. Material and methods: An exploratory, descriptive cross-sectional study of a quantitative nature, conducted with 162 patients from August to October 2018. Hypertensive patients, over 18 years of age characterized by a semi-structured interview, were included in the questionnaire. in households. The data tabulation was done in Excel 2016 worksheet, and presented in the form of tables with absolute and relative frequencies. Results: The largest contingent of respondents were women (65\%) and elderly, between 59 and over 65 (73\%), with a low educational level (70\%). Diabetes $(27 \%)$ and sedentary lifestyle (58\%) were the most prevalent risk factors. The female population had a higher rate of abdominal obesity (48\%). Conclusion: The hypertensive profile of this unit is comprised mostly of female patients, advanced age and presenting risk factors for the development of cardiovascular diseases that deserve greater assistance. The acquired information can contribute to the health team to know better its population and to develop strategies of interventions about the treatment and accompaniment to control the disease and to improve the quality of life of the same.

Descriptors: Hypertension. Cardiovascular diseases. Risk factors.

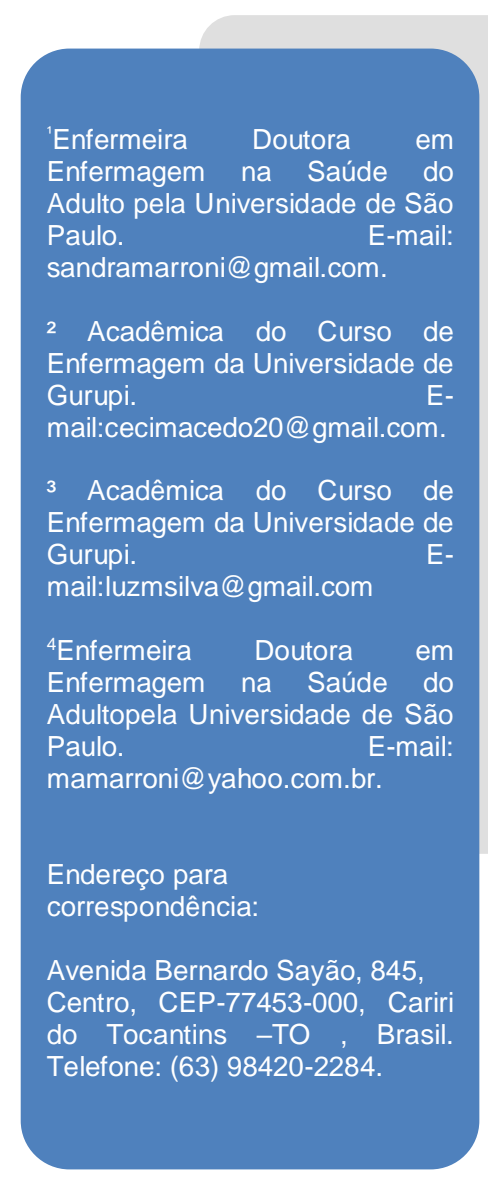




\section{INTRODUCAO}

A Hipertensão Arterial (HA) é uma condição clínica multifatorial, caracterizada por níveis elevados e sustentados de pressão arterial $\geq 140 \mathrm{e} / \mathrm{ou} 90 \mathrm{mmHg}$, que apresenta grande risco de desenvolvimento de Doenças Cardiovasculares - DCV e lesões em órgãos-alvo como encéfalo, rins, coração e vasos, com alta taxa de mortalidade e a alterações metabólicas com consequente aumento do risco de eventos cardiovasculares fatais e não fatais. 1

Além de a hipertensão arterial ser um dos mais importantes fatores de risco para doenças cardiovasculares, merece atenção especial os demais fatores não menos importantes e que também estão intimamente ligados com as DCV, destacando-se o tabagismo, etilismo, estresse, sedentarismo, obesidade, consumo excessivo de sódio, e baixo nível de escolaridade.1-4 A hipertensão agrava-se na presença desses fatores, e principalmente quando há dislipidemia, obesidade abdominal, intolerância à glicose e diabetes.1 A presença de diabetes é pelo menos duas vezes mais prevalente em hipertensos do que na população em geral e são condições que favorecem complicações da mesma, bem como as dislipidemias que são caracterizadas pela presença de níveis alterados de colesterol (total e/ou frações) e de triglicérides no organismo. Portadores desses distúrbios metabólicos possuem risco elevado para doença cardiovascular quando comparados àqueles que não o possuem, sendo que o risco individual dependente da associação com outros fatores de risco. 5

A prevalência da hipertensão estima-se aproximadamente de 30 a $45 \%$ na população em geral, com aumento acentuado relacionado com o envelhecimento.6Estudo realizado em 2016 mostrou que houve aumento de 3\% da população brasileira. Em 2010 a prevalência era de 195.497 .797 brasileiros, e foi para 204.450 .649 brasileiros em 2015, com percentual de $49 \%$ do sexo masculino e $51 \%$ do sexo feminino. Houve envelhecimento da população brasileira neste período, com aumento de $22 \%$ da população acima de 65 anos.7 Há associação direta entre envelhecimento e prevalência de hipertensão arterial, relacionada ao aumento da expectativa de vida da população brasileira e aumento na população de idosos.1 Do total de óbitos no Brasil, as DCV representaram $28 \%$. Houve declínio no número de internações clínicas por esta doença de 874.949 no ano de 2010, para 807.304 no ano de 2015. Porém, houve um crescente número de internações cirúrgicas por DCV de 246.038 para 279.010, gerando altos custos para os cofres públicos.7 
Em onze anos, o diagnóstico médico de hipertensão arterial aumentou na população adulta das capitais brasileiras e Distrito Federal. De acordo com o Sistema de Vigilância de Fatores de Risco e Proteção para Doenças Crônicas por Inquérito Telefônico (VIGITEL) de 2017, maior estudo de prevalência de hipertensão autorreferida realizada no Brasil desde 2006, nas 26 capitais e Distrito Federal, passou de 22,6\% em 2006 para $24,3 \%$ em 2017. A doença tende a aumentar com a idade, chegando, em 2017, a 60,9\% entre os adultos com 65 anos e mais; foi menor entre aqueles com maior escolaridade, com 14,8\% entre aqueles com 12 anos ou mais de estudo. De acordo com o estudo, as mulheres ainda continuam com maior prevalência de diagnóstico médico de hipertensão arterial quando comparado aos homens, tendo registrado $26,4 \%$ contra $21,7 \%$ para eles.

Em 2017, as capitais com maior prevalência entre as mulheres foram Rio de Janeiro (34,7\%) e Recife (30,0\%), e entre os homens, foram Maceió $(26,3 \%)$ e Natal $(26,2 \%)$. A capital brasileira com maior incidência total de hipertensão arterial, por dois anos consecutivos foi o Rio de janeiro com 30,7\%.9

O tratamento da hipertensão consiste na mudança de estilo de vida, dieta adequada pobre em gorduras e baixo nível de sódio, associada a prática regular de exercícios físicos e uso de medicamentos para manter os níveis pressóricos, buscando reduzir a morbimortalidade. A terapêutica medicamentosa adequada se dá através da avaliação do estágio da doença, porém, deve ser sempre associada às práticas saudáveis para obter melhores resultados. 5

Recomenda-se, que os pacientes sejam acompanhados por equipe multidisciplinar e que a família esteja envolvida, o que pode contribuir para maior adesão e melhores chances de sucesso com o tratamento. A adesão pode ser caracterizada, como grau em que o comportamento do indivíduo, em termos de tomar o medicamento, seguir dieta, realizar mudanças de estilo de vida e comparecer às consultas, interfere e contribui em seu tratamento e também pelo grau de coincidência entre a prescrição médica e o comportamento do paciente. 10

Para pacientes com níveis inadequados da pressão arterial são indicadas avaliações médicas mensais no intuito de obter a meta pressórica adequada o quanto antes possível se necessário, ajustando-se as medidas terapêuticas. As consultas de avaliação são recomendadas até obter o nível desejável da pressão arterial, mensais para pacientes com hipertensão moderada, e mais freqüentes para hipertensão grave. Depois de alcançados os níveis esperados, as consultas de controle podem ser trimestrais ou 
semestrais. É importante que o tratamento seja seguido corretamente e os pacientes sejam acompanhados regularmente para observar se os níveis tensionais estão adequados, para possíveis intervenções necessárias a fim de diminuir o risco de complicações e controlar a doença. 1

Sendo assim, este estudo tem como objetivo conhecer o perfil desses hipertensos, em relação às características biossociais, tratamento, hábitos e estilo de vida e alguns riscos para desenvolvimento de doença cardiovascular através de medidas antropométricas.

Descrever pontos importantes do perfil desta clientela possibilita colaborar com a assistência e conhecimento da equipe quanto aos hábitos e atributos deste grupo.

\section{MATERIAIS E METODOS}

Trata-se de um estudo exploratório, descritivo transversal, de natureza quantitativa efetivada por meio de entrevista com elemento semiestruturado. A pesquisa foi realizada na USF Manoel Pedro Pires Filho, situada no município de Cariri do Tocantins localizado ao sul do estado, às margens da BR-153, no sentido Belém - Brasília.

Participaram da pesquisa os pacientes que se encaixaram nos critérios de inclusão: idade $\geq 18$ anos, hipertensos cadastrados na USF e no e-SUS, residentes na zona urbana e que aceitaram participar do estudo por meio de assinatura do Termo de Consentimento Livre e Esclarecido, sendo excluídos os acamados e portadores de distúrbios mentais. A amostra final obtida foi calculada considerando uma margem de erro de $5 \%$ para mais ou para menos. Igualmente foi utilizado um cálculo de amostragem simples resultando em 162 pacientes da amostra de Barbetta. Foi selecionada a quantidade correspondente à cada uma das 7 micro áreas atendidas pela Unidade de Saúde Manoel Pedro Pires Filho do município de Cariri do Tocantins.12As propostas foram apresentadas à Secretária Municipal de Saúde do município de Cariri, Maria Auxiliadora da Paixão Aires, a qual concordou com a realização da pesquisa. $\mathrm{O}$ projeto foi submetido à plataforma Brasil onde obtivemos aprovação do comitê de ética e pesquisa da Universidade de Gurupi, sob o parecer $n^{\circ}$ 2.832.200/2018.

A fim de alcançar o objetivo foi elaborado questionário a partir de instrumentos já utilizados em outros estudos, com questões objetivas acerca de dados biossociais, utilização de medicamentos, comparecimento nas consultas e grupos de apoio, hábitos e estilo de vida, como tabagismo, etilismo, prática de exercícios físicos, alimentação, doenças associadas, e coleta de dados antropométricos para obtenção de IMC -índice de 
massa corporal - e circunferência abdominal. $O$ instrumento foi aplicado pelas pesquisadoras na Unidade de Saúde e nos domicílios dos participantes selecionados acompanhadas dos agentes comunitários de saúde, até se obter o tamanho da amostra instituída de acordo com os critérios acima descritos.

Para realização das medidas antropométricas foi utilizada balança portátil digital da marca Omron Hn 289 LA com limite de até $150 \mathrm{~kg}$, apropriada para uso em pesquisas, calibrada para a medida do peso. Além de uma fita métrica para medir a estatura e a circunferência abdominal em centímetros. O IMC foi calculado pela razão peso/altura2 $(\mathrm{kg} / \mathrm{m} 2)$. A classificação utilizada foi: magro ou baixo peso $(<18,5)$, normal ou eutrófico (18,5-24,9), sobrepeso ou pré-obeso (25-29,9), obesidade I (30-34,9), obesidade II (30$39,9)$, obesidade III grave ou mórbida $(\geq 40,0)$ e o ponto de corte da circunferência abdominal deve ser de $102 \mathrm{~cm}$ para homens e $88 \mathrm{~cm}$ para mulheres de acordo com as Diretrizes Brasileiras de Obesidade 2016/ABESO.13

Segundo a Organização Mundial da Saúde - OMS, a circunferência abdominal é a medida da região do abdômen, no ponto médio entre a borda inferior da última costela e a borda superior da crista ilíaca, onde pode se concentrar a gordura visceral, diretamente relacionada a diversos riscos como aumento da pressão arterial, diabetes e colesterol alto, fatores que elevam o risco de DCV.14

Para a tabulação dos dados as informações adquiridas foram inseridas em planilha do Excel 2016, para realizar a contagem das informações. Feito isso os resultados serão apresentados em forma de freqüências absolutas e relativas, exibidos em tabelas discutidas no decorrer do estudo.

\section{RESULTADOS E DISCUSSÁO}

Os resultados expressos na Tabela 1 mostram que maior parte dos hipertensos é do sexo feminino (65\%). De acordo com estudos realizados, as mulheres buscam mais comumente tratamento médico, sendo prontamente diagnosticadas, o que as expõe menos a riscos e após os 45-50 anos de idade são mais acometidas pela hipertensão devido à pós-menopausa com baixa do estrógeno que tem efeito cardioprotetor. ${ }^{8,15,16}$ 
Tabela 1 - Características biossociais dos hipertensos da USF Manoel Pedro Pires Filho.

\begin{tabular}{|c|c|c|}
\hline \multirow[t]{2}{*}{ Variáveis } & \multicolumn{2}{|c|}{ Respostas } \\
\hline & $\mathbf{N}$ & $\%$ \\
\hline \multicolumn{3}{|l|}{ Idade } \\
\hline $18-25$ & 0 & $0 \%$ \\
\hline $26-30$ & 1 & $1 \%$ \\
\hline $31-35$ & 4 & $2 \%$ \\
\hline $36-40$ & 6 & $4 \%$ \\
\hline $41-45$ & 12 & $7 \%$ \\
\hline $46-50$ & 11 & $7 \%$ \\
\hline $51-55$ & 10 & $6 \%$ \\
\hline $56-60$ & 23 & $14 \%$ \\
\hline $61-65$ & 25 & $15 \%$ \\
\hline$>65$ & 70 & $43 \%$ \\
\hline \multicolumn{3}{|l|}{ Gênero } \\
\hline Fem. & 105 & $65 \%$ \\
\hline Masc. & 57 & $35 \%$ \\
\hline \multicolumn{3}{|l|}{ Etnia } \\
\hline Branco & 70 & $43 \%$ \\
\hline Negro & 35 & $22 \%$ \\
\hline Pardo & 56 & $35 \%$ \\
\hline Amarelo & 1 & $1 \%$ \\
\hline \multicolumn{3}{|l|}{ Estado civil } \\
\hline Solteiro & 15 & $9 \%$ \\
\hline Casado & 90 & $56 \%$ \\
\hline Amasiado & 11 & $7 \%$ \\
\hline União estável & 2 & $1 \%$ \\
\hline Divorciado & 17 & $10 \%$ \\
\hline Viúvo & 27 & $17 \%$ \\
\hline \multicolumn{3}{|l|}{ Escolaridade } \\
\hline Não alfabetizado & 20 & $12 \%$ \\
\hline Lê-escreve & 24 & $15 \%$ \\
\hline Ens. Fundamental incompleto & 69 & $43 \%$ \\
\hline Ens. Fundamental completo & 11 & $7 \%$ \\
\hline Ens. Médio incompleto & 11 & $7 \%$ \\
\hline Ens. Médio completo & 19 & $12 \%$ \\
\hline Ens. Sup. Completo & 8 & $5 \%$ \\
\hline Total & 162 & $100 \%$ \\
\hline
\end{tabular}

Fonte:Autoria própria, dados da pesquisa.

A idade prevalente foi entre 56 e mais que 65 anos totalizando (72\%) da população estudada, o que está em evidência em estudo citado anteriormente, no qual a população idosa é a que mais apresenta condição clínica devido ao aumento da expectativa de vida $>60$ anos e a crescente da população idosa. ${ }^{1,15}$

Quanto à etnia: 43\% de cor branca; 35\% negros; 22\% pardos. Em outro estudo semelhante os não brancos predominaram, neste estudo totalizaram juntos $57 \%{ }^{15}$

Quanto ao estado civil $56 \%$ são casados, 17\% viúvos, 10\% divorciados, 9\% solteiros, $7 \%$ amasiados e $9 \%$ solteiros, como em outro estudo realizado no qual em relação ao estado civil, prevaleceu o casado, seguido da viuvez. ${ }^{17}$ 
Observa-se a baixa escolaridade, visto que $43 \%$ não concluíram o ensino fundamental, $12 \%$ são analfabetos e $15 \%$ lê-escreve. Apenas $5 \%$ chegaram a concluir o ensino superior. Estudos também revelam que a pressão arterial tende a elevar-se nos pacientes com menor grau de escolaridade. ${ }^{18-20}$ Outro estudo mostrou que em $21 \%$ do total dos hipertensos estudados a hipertensão arterial foi maior em pacientes com baixa escolaridade. ${ }^{21}$ Níveis baixos de instrução estão relacionados à incidência da doença, devido esta população geralmente apresentar pouco entendimento sobre a doença, podendo interferir na adesão ao tratamento. ${ }^{1}$

Tabela 2 - Características quanto ao tratamento medicamentoso dos hipertensos da USF Manoel Pedro Pires Filho.

\begin{tabular}{lcc}
\hline \multicolumn{1}{c}{ Variáveis } & Respostas \\
\hline Tratamento medicamentoso & $\mathbf{N}$ & $\%$ \\
Monoterapia & 101 & $62 \%$ \\
2 & 43 & $27 \%$ \\
3 ou mais & 18 & $11 \%$ \\
\hline Total & $\mathbf{1 6 2}$ & $\mathbf{1 0 0 \%}$ \\
\hline
\end{tabular}

Fonte: Autoria própria, dados da pesquisa.

Os dados da Tabela 2 mostram que a maioria dos pacientes realiza tratamento com apenas uma medicação: monoterapia (62\%). 27\% utilizam dois tipos de medicação e $11 \%$ necessitam de 3 ou mais medicamentos. O tratamento medicamentoso pode ser realizado com uma ou mais classes de fármacos, de acordo com a necessidade, para que sejam alcançadas as metas para a pressão arterial e de acordo com situações específicas. A monoterapia pode ser a estratégia inicial para pacientes com hipertensão estágio 1, com risco cardiovascular baixo e moderado. Não obstante, deve-se observar que, de acordo com a meta a ser atingida, a maioria dos pacientes poderá necessitar da associação de medicamentos. O tratamento deve ser individualizado e a escolha inicial do medicamento a ser utilizado deve ser baseada em alguns aspectos como: capacidade de o agente escolhido reduzir a morbimortalidade cardiovascular; mecanismo fisiopatogênico predominante no paciente a ser tratado; características individuais; doenças associadas; condições socioeconômicas. 
Tabela 3 - Condutas associados às medidas facilitadoras para o tratamento dos hipertensos da USF Manoel Pedro Pires Filho.

\begin{tabular}{lcc}
\hline \multicolumn{1}{c}{ Variáveis } & Respostas & \\
\hline Usa a medicação todos os dias nos & $\mathbf{N}$ & $\%$ \\
mesmoshorários? & 139 & $86 \%$ \\
Sim & 10 & $6 \%$ \\
Não & 13 & $8 \%$ \\
Às vezes & 23 & $14 \%$ \\
Deixa de tomar quando está se sentindo bem? & 131 & $81 \%$ \\
Sim & 8 & $5 \%$ \\
Não & & \\
Às vezes & 135 & $83 \%$ \\
Comparece às consultas agendadas & 13 & $8 \%$ \\
periodicamente? & 14 & $9 \%$ \\
Sim & & \\
Não & 48 & $30 \%$ \\
Às vezes & 89 & $55 \%$ \\
Frequenta o grupo de apoio (Hiperativo)? & 25 & $15 \%$ \\
Sim & $\mathbf{1 6 2}$ \\
Não & $\mathbf{1 0 0 \%}$ \\
Às vezes & & \\
Total &
\end{tabular}

Fonte:Autoria própria, dados da pesquisa.

Na Tabela 3 percebe-se que ao serem questionados se faziam uso da medicação diariamente, $86 \%$ dos pacientes alegaram que sim, o quedemonstra o uso correto da medicação. Alguns pacientes, ao não perceberem nenhum sintoma característico da elevação da pressão arterial deixam de tomar a medicação (14\%), porém a grande maioria entende que necessitam fazer o uso contínuo (81\%) independente de não apresentar ou não mal-estar devido à doença. Estudo desenvolvido em um Ambulatório de Hipertensão e Metabologia sobre adesão ao tratamento medicamentoso revelou que a adesão esteve presente em $87 \%$ dos pacientes estudados. ${ }^{19}$ Compreender que devem tomar correta e continuamente a medicação contribui para maior sucesso do tratamento e controle dos níveis tensionais.

Quanto ao comparecimento às consultas de rotina, 83\% responderam que comparecem à Unidade de Saúde quando solicitados e para realizar o acompanhamento periodicamente. Estudo publicado em 2018 refere que maior parte dos hipertensos comparece às unidades para as consultas, revelando que apenas $11,1 \%$ relataram não ter utilizado consultas médicas disponíveis. Os resultados deste estudo permitiram verificar que, apesar de ter sido baixa a prevalência de não utilização das consultas de rotina, homens, indivíduos de cor não branca e que utilizavam apenas os serviços do Sistema Único de Saúde, exibiram maior chance de não buscarem este tipo de atendimento na atenção básica. ${ }^{22}$ 
A alta taxa de comparecimento em consultas e utilização correta diária da medicação pode também ser reflexo de uma ação recente da equipe da unidade que realizou uma busca ativa dos pacientes fornecendo-lhes um cartão de agendamento de retorno e consultas. O cartão contém ainda as medicações em uso e dados alusivos à Hipertensão e alguns fatores de risco, o que confirma que a adesão ao tratamento está diretamente ligada às ações realizadas pela equipe multidisciplinar para incentivar o paciente a seguir adequadamente o tratamento e estar à par de sua situação clínica.

Referente à participação no grupo de apoio (Grupo Hiperativo) apenas 30\% dos entrevistados afirmam participar e 55\%, sendo a maioria, não participam dos encontros do grupo. Os grupos de apoio têm o objetivo de fornecer informações e recreação para os pacientes, visando sempre maiores taxas de adesão ao tratamento dessa clientela. Ressaltando ainda que a adesão ao tratamento compreende a relação entre a prescrição médica e o comportamento do paciente em seguir dieta adequada, realizar mudança de estilo de vida, como abandono do tabagismo, etilismo e sedentarismo e tomada correta da medicação. ${ }^{10}$

A fim de caracterizar os hábitos e estilo de vida dos hipertensos, a Tabela 4 demonstra que apenas 34\% alegaram praticar exercícios físicos regularmente, e 58\% não o fazem. Estudo semelhante realizado em Ananindeua-PA, revelou que apenas 14,52\% dos hipertensos entrevistados referiram praticar algum tipo de atividade física, os baixos índices reforçam a ideia da dificuldade dessa população em abandonar o sedentarismo. ${ }^{23}$ O exercício físico regular reduz a pressão arterial sistólica e diastólica em normotensos, o que justifica sua prática. ${ }^{5}$ Assim, a prática regular de exercícios físicos pode auxiliar na redução dos níveis pressóricos, devendo ser integrado ao tratamento.

Tabela 4 - Hábitos e estilo de vida dos hipertensos da USF Manoel Pedro Pires Filho.

\begin{tabular}{lcc}
\hline \multicolumn{1}{c}{ Variáveis } & Respostas \\
\hline Pratica exercícios físicos regularmente? & $\mathbf{N}$ & $\%$ \\
Sedentarismo & & \\
Sim & 55 & $34 \%$ \\
Não & 94 & $58 \%$ \\
Às vezes & 13 & $8 \%$ \\
Tabagismo & 26 & $16 \%$ \\
Sim & 135 & $83 \%$ \\
Não & 1 & $1 \%$ \\
Às vezes & & \\
Tem o hábito de ingerir bebida alcoólica mais & 6 & $4 \%$ \\
de 2x por semana? & 145 & $90 \%$ \\
Sim & 11 & $7 \%$ \\
Não & &
\end{tabular}




\begin{tabular}{lcc} 
Você se considera uma pessoa estressada? & & \\
Sim & 53 & $33 \%$ \\
Não & 81 & $50 \%$ \\
Às vezes & 28 & $17 \%$ \\
Tem o hábito de ingerir com frequência fritura, & & \\
doces, refrigerantes? & 49 & $30 \%$ \\
Sim & 76 & $47 \%$ \\
Não & 37 & $23 \%$ \\
Às vezes & & $7 \%$ \\
Tem o hábito de adicionar sal à comida pronta? & 12 & $93 \%$ \\
Sim & 150 & $\mathbf{1 0 0} \%$ \\
Não & $\mathbf{1 6 2}$ \\
\hline Total &
\end{tabular}

Fonte:Autoria própria, dados da pesquisa.

O tabagismo tem baixa taxa, já $83 \%$ não fumam e $16 \%$ fazem uso do tabaco. Poucos entrevistados afirmaram ter o hábito de ingerir bebida alcoólica mais de duas vezes por semana (4\%) e a maioria expressiva (90\%) não faz uso de álcool, sendo tais atitudes fatores protetivos para os mesmos, pois tantoo tabagismo quanto o etilismo propiciam o aumento de doenças cardiovasculares. No primeiro, o etanol estabelece um efeito deletério sobre o sistema cardiovascular, provocando a elevação dos níveis tensionais, particularmente da pressão sistólica e prevalência de hipertensão arterial. No segundo, a nicotina estimula a liberação da norepinefrina das terminações nervosas adrenérgicas. Adicionalmente, a nicotina e outros componentes do cigarro lesam o endotélio dos vasos sanguíneos, provocando arteriosclerose, o que diminui a complacência vascular e propicia o aumento dos níveis tensionais, levando a maior incidência das doenças coronária, cerebrovascular e vascular de extremidade. ${ }^{24-5}$

Em relação ao estresse, metade dos entrevistados (50\%) não se considera estressado, 33\% alegaram que sim e 17\% às vezes se sentem estressados. $\mathrm{O}$ estresse pode ser de natureza física, psicológica ou social. Sobrecarga deste fator de risco pode aumentar os danos à saúde do indivíduo, agravar uma patologia já existente ou facilitar o aparecimento da mesma se o indivíduo já possuir predisposição para o desenvolvimento da patologia. ${ }^{25}$

A alimentação contendo doces, frituras, refrigerantes e enlatados foi afirmada por $30 \%$, $23 \%$ sendo somente às vezes e $47 \%$ não tinham o hábito de ingerir esse tipo de alimento, neste quesito percebe-se que os entrevistados se dividemem seguir uma dieta balanceada e saudável. O sucesso do tratamento está ligado com medidas nutricionais e depende da adoção de um plano alimentar saudável e sustentável. Algumas medidas recomendadas para alcançar uma dieta saudável incluem ingestão de frutas, legumes, vegetais, peixes, diminuição do consumo de sal, doces e gordura. ${ }^{1}$ 
O costume de adicionar sal à comida pronta foi referido por $7 \%$, enquanto $93 \%$ não o fazem. Este é um hábito que não deve ser praticado e a alimentação deve conter baixo nível de sal. O consumo excessivo de sódio está associado ao aumento progressivo da pressão arterial, e, por conseguinte aumento no risco de doenças cardiovasculares, que são as principais causas de morte no Brasil e no mundo. A quantidade de sódio disponível para consumo nos domicílios brasileiros é duas vezes maior que o limite máximo de ingestão recomendado pela Organização Mundial de Saúde. A disponibilidade demasiada de sódio continua sendo observada em todas as regiões do país, nos meios urbanos e rurais e em todas as classes de renda. A disponibilidade domiciliar no Brasil foi de 4,7 g/pessoa/dia, ajustada para consumo de $2.000 \mathrm{kcal}$, ultrapassando em mais de duas vezes o consumo máximo preconizado de $2 \mathrm{~g} / \mathrm{dia}$. Embora a maioria do sódio seja derivado do sal de cozinha e condimentos, alimentos processados apresentam alta concentração do mesmo, chegando a $25 \%$ do total consumido. ${ }^{26}$

Comorbidades associadas estão presentes em $46 \%$ dos entrevistados, dentre elas a diabetes (27\%) como mais prevalente, seguido das dislipidemias (22\%), que são fatores de risco para o agravamento da hipertensão arterial, bem como para desenvolver doenças cardiovasculares. ${ }^{1-5}$

Tabela 5. Dados antropométricos e alguns fatores de risco para doença cardiovascular dos hipertensos da USF Família Manoel Pedro Pires Filho.

\begin{tabular}{lcc}
\hline \multicolumn{1}{c}{ Variáveis } & Respostas \\
\hline Outras doenças & $\mathbf{N}$ & $\%$ \\
Não & 88 & $54 \%$ \\
Sim & 74 & $46 \%$ \\
Doenças & 36 & $22 \%$ \\
Dislipidemias & 43 & $27 \%$ \\
Diabetes & 1 & $1 \%$ \\
Infarto & 9 & $6 \%$ \\
Outra & & $2 \%$ \\
IMC & 3 & $17 \%$ \\
Baixo peso $(<18,5)$ & 28 & $19 \%$ \\
Normal $(18,5-24,9)$ & 30 & $11 \%$ \\
Obesidade I $(30-34,9)$ & 18 & $8 \%$ \\
Obesidade II (35-39,9) & 13 & $43 \%$ \\
Obesidade III $(>40,0)$ & 70 & $17 \%$ \\
Sobrepeso $(25-29,9)$ & & $48 \%$ \\
Circunferência abdominal Feminino & 27 & \\
$<88 \mathrm{~cm}$ & 78 & $22 \%$ \\
$>88 \mathrm{~cm}$ & & $14 \%$ \\
Circunferência abdominal Masculino & 35 & $\mathbf{1 0 0 \%}$ \\
\hline $102 \mathrm{~cm}$ & 22 & \\
\hline $102 \mathrm{~cm}$ & $\mathbf{1 6 2}$ & \\
\hline Total & &
\end{tabular}

Fonte: Autoria própria, dados da pesquisa. 
A coleta de dados antropométricos (peso e altura) foi realizada buscando identificar a existência para risco cardiovascular e peso acima do recomendado entre a população estudada. Quanto ao IMC, 43\% estão com sobrepeso (pré-obesos), a soma de pacientes com algum grau de obesidade chega a $38 \%$, na qual $19 \%$ apresentam obesidade grau I, $11 \%$ obesidade grau II e $8 \%$ obesidade grau III, e apenas $17 \%$ estão com o peso dentro do preconizado.Estudo realizado revelou que $39 \%$ de uma população apresentavam morbidades relacionadas, predominando entre elas a Diabetes Melitus. O mesmo estudo revela que $69 \%$ dos entrevistados se encontravam obesos, sendo este um fator de risco para doenças cardiovasculares. Os dados de IMC demonstram que $28 \%$ dos hipertensos entrevistados apresentaram obesidade leve, 39\% possuíam obesidade elevada e $2 \%$ obesidade mórbida. Tais dados são preocupantes, uma vez que o excesso de peso aumenta de duas a seis vezes o risco de hipertensão arterial. A má distribuição da gordura corporal, com deposição excessiva abdominal, pode ser usada como um indicador de risco para doenças cardiovasculares. ${ }^{20}$

Outro estudo realizado no conjunto habitacional metropolitano, da cidade de Caucaia, área metropolitana de Fortaleza, indica que a população estudada apresentou uma alta prevalência de excesso de peso e de hipertensão arterial, sendo que a ocorrência de hipertensão arterial esteve fortemente associada ao excesso de peso, fator apresentado por $51,26 \%$, e de hipertensão arterial $22,58 \%{ }^{27}$

Em relação à Circunferência Abdominal - CA entre as mulheres, 17\% estão dentro da normalidade, enquanto que $48 \%$ apresentam CA $>88 \mathrm{~cm}$. Os homens têm índices menores em comparação às mulheres: $22 \%$ estão no parâmetro aceitável e $14 \%$ CA > 102. Os resultados são semelhantes aos de estudo realizado em um serviço ambulatorial (Liga de Hipertensos) de um hospital de ensino da cidade de São Paulo, no qual em relação medida da cintura, 33\% dos homens tinham cintura maior que $102 \mathrm{~cm}$ e $74 \%$ das mulheres maior que $88 \mathrm{~cm}$, que são os limites máximos tolerados. ${ }^{28} \mathrm{Em}$ uma USF do município de Londrina, Paraná, a prevalência de obesidade abdominal identificada pela circunferência abdominal foi maior no sexo feminino $82,8 \%$ enquanto que $36,0 \%$ dos homens não estava com a CA adequada. ${ }^{29}$ Outro estudo realizado, desta vez apenas com mulheres revelou que aquelas que apresentam circunferência abdominal acima do recomendado tem 2 vezes mais chances de ter hipertensão do que as que não apresentam obesidade abdominal. ${ }^{30}$ 


\section{CONSIDERAÇOES FINAIS}

O perfil dos pacientes pesquisados possui características comuns e muito citadas em demais estudos com essa população. A pesquisa apresentou prevalência do sexo feminino, idosos e com baixa escolaridade. O sedentarismo e doenças associadas mais referidas foram a diabetes e dislipidemias, sendo estes fatores de risco altamente prevalentes na hipertensão arterial e nas doenças cardiovasculares.

As características dos hipertensos dessa USF estão em consonância com publicações existentes, apesar de se tratar de uma cidade pequena e sem muitas opções e diversidade de serviços de saúde,algumas variáveis estão dentro da normalidade como o seguimento adequado do tratamento medicamentoso e assiduidade em consultas de rotina. Apesar de este aspecto apresentar satisfação, o sedentarismo ainda é um problema enfrentado pelos hipertensos, pois os mesmos não conseguem manter uma rotina com prática de exercícios físicos recomendados. O estudo pode ter sofrido interferências levando em consideração que as respostas foram auto referidas pelos entrevistados, porém dentro da margem de erro considerada na amostra, de 5\% para mais ou para menos.

Diante do exposto, convém ressaltar a necessidade constante de criar e adotar políticas de saúde adequadas para manter bons resultados e promover o controle da patologia, visando melhorar a qualidade de vida dessa clientela, evitando comorbidades associadas. Neste contexto a enfermagem tem um papel importante a desempenhar na assistência prestada a esse público, fornecendo informações e elaborando estratégias adequadas de intervenções. Por isso é imprescindível que a equipe multidisciplinar conheça bem sua comunidade para que possam prestar assistência individualizada e eficaz buscando a recuperação, promoção e prevenção da saúde.

\section{REFERENCIAS}

\footnotetext{
${ }^{1}$ Malachias MVB, Souza WKSB, Plavnik FL, Rodrigues CIS, Brandão AA, Neves MFT, Bortolotto LA, etal. 7 a Diretriz Brasileira de Hipertensão Arterial. Arq Bras Cardiol. 2016 [acesso em 2019 abr 29]; 107(3):1-83. Disponível em:http://publicacoes.cardiol.br/2014/diretrizes/2016/05_HIPERTENSAO_ARTERIAL.pdf.

2Williams B. The year in hypertension. JACC. 2010[acesso em 2019 abr 29]; 55(1): 66-73. Disponível em:https://www.sciencedirect.com/science/article/pii/S0735109708010322?via\%3Dihub.

3Passos VMA et al. Hipertensão no Brasil: estimativa de prevalência a partir de estudos de base populacional. Epidemiol Serv Saúde. 2006 [acesso em 2019 abr 29], 15(1): 35-45. Disponível em: http://scielo.iec.gov.br/scielo.php?script=sci_abstract\&pid=S1679-49742006000100003\&lng=pt\&nrm=is.
} 
4Jardim PCV et al. Hipertensão Arterial e alguns Fatores de Risco em uma capital brasileira. Arq Bras Cardiol. 2007 [acesso em 2019 abr 29]; 88(4):452-57. Disponível em: http://www.scielo.br/scielo.php?pid=S0066782X2007000400015\&script=sci_abstract\&tlng=pt.

5Sociedade Brasileira de Hipertensão (SBH). I Diretriz brasileira de diagnóstico e tratamento da síndrome metabólica: tratamento medicamentoso da dislipidemia. Rev Soc Bras Hipert. 2004 [acesso em 2019 abr 29]; 7(4):143-45. Disponível em: http://www.scielo.br/pdf/abc/v84s1/a01v84s1.pdf.

$62013 \mathrm{ESH} / \mathrm{ESC}$ Guidelines for the management of arterial hypertension, TheTask Force for the management ofarterial hypertension of the European Society of Hypertension (ESH) and of the European Society of Cardiology (ESC). Journal of Hypertension. 2013 [acesso em 2019 abr 29], 31(7):1281-1357. Disponível em: https://www.sphta.org.pt/files/esh_esc_guidelines_2013.pdf.

7Siqueira ASP, Siqueira Filho AG, Land MGP. Análise do Impacto Econômico das Doenças Cardiovasculares nos Últimos Cinco Anos no Brasil. Arq Bras Cardiol. 2017[acesso em 2019 abr 29]; 109(1): 39-46. Disponível

http://www.scielo.br/scielo.php?pid=S0066782X2017005009101\&script=sci_arttext\&tlng=pt.

8Instituto Brasileiro de Geografia e Estatística. Pesquisa Nacional de saúde 2013: percepção do estado de saúde, estilos de vida e doenças crônicas: Brasil, Grandes Regiões e Unidades da Federação.2013[acesso em 2019 abr 29]. Disponível em: http://www.ibge.gov.br/home/estatistica/populacao/pns/2013/default.shtm.

9Ministério da Saúde. Vigitel Brasil 2017. Vigilância de fatores de risco e proteção para doenças crônicas por inquérito telefônico. 2017 [acesso em 2019 abr 29]. Disponível em: http://bvsms.saude.gov.br/bvs/publicacoes/vigitel_brasil_2017_vigilancia_ fatores_riscos.pdf.

10Pierin AMG. Aspectos conceituais. In: Nobre F, Mion DJ. Adesão ao tratamento o grande desafio da hipertensão. São Paulo: Lemos Editorial; 2001.

11CervoAL et al. Metodologia científica. 6 ed. São Paulo: Pearson Prentice Hall, 2007

12Barbetta, PA. Estatística Aplicada às Ciências Sociais. 8ªed., Florianópolis: UFSC, 2012.

13Associação Brasileira para o Estudo da Obesidade e da Síndrome Metabólica Diretrizes brasileiras de obesidade. 4.ed. São Paulo: ABESO, 2016.

14Portal do Ministério da saúde. Só o IMC não diz como você está. 2017 [acesso em 2019 abr 29]. Disponível em: http://portalms.saude.gov.br/component/content/article/804-imc/40508-so-o-imc-nao-dizcomo-voce-esta.

15Correia BR, Ribeiro DF, Carvalho QGS,Machado ALG, Douberine CA, Gubert FA, Vieira NFC. Perfil Clínico-Epidemiológico de Pacientes Assistidos em Clínica de Hipertensão. JHealth Sci. 2017 [acesso em 2019 29];19(2):171-6. Disponível em: http://revista.pgsskroton.com.br/index.php/JHealthSci/article/view/4647.

16Carlos PR, Palha PF, Veiga EV, Beccaria LM. Perfil de hipertensos em um núcleo de saúde da família. Arq Ciênc Saúde 2008 [acesso em 2019 abr 29];15(4):176-81. Disponível em: http://repositorioracs.famerp.br/racs_ol/vol-15-4/IDB\%20296.pdf.

17Bezerra ASM, Lopes JL,Barros ALBL. Adesão de pacientes hipertensos ao tratamento medicamentoso. Rev Bras Enferm. 2014 [acesso em 2019 abr 29];67(4):550-5. Disponível em: http://dx.doi.org/10.1590/0034-7167.2014670408.

18Sanches, CG, Pierin AMG. Comparação dos perfis dos pacientes hipertensos atendidos em ProntoSocorro e em tratamento ambulatorial. Rev Esc Enf USP 2004 [acesso em 2019 abr 29]; 38(1): 90-8. Disponível em: http://www.scielo.br/pdf/reeusp/v38n1/11.pdf.

19Cabral AH. Caracterização dos hipertensos atendidos nas unidades básicas de saúde da cidade de Sorocaba [dissertação]. São Paulo: Escola de Enfermagem da USP; 2000. 
DOI 10.18606/2318-1419/amazonia.sci.health.v7n2p55-69
Marroni SN, Macedo JC, Silva LM, Marroni MA

Características de um Grupo de Hipertensos em uma USF da Cidade Cariri do Tocantins

20Cordeiro BR. A pressão arterial em funcionários do hospital universitário regional do norte do Paraná: associação das variáveis biopsicossociais, hábitos de vida econhecimento sobre hipertensão [dissertação]. São Paulo: Escola de Enfermagem da USP; 2000.

21Fuchs FD. Moreira WD, Ribeiro JP. Efeitos do exercício físico na prevenção e tratamento da hipertensão arterial: avaliação por ensaios clínicos randomizados. Hipertensão; 2001; 4:91-3.

22Barreto MS, Mendonça RD, Pimenta AM, Garcia-Vivar C,Marcon SS. Não utilização de consultas de rotina na Atenção Básica por pessoas com hipertensão arterial. Ciênc saúde colet. 2018[acesso em 2019 abr 29]; 23 (3): 795-804. Disponível em: https://www.scielosp.org/article/csc/2018.v23n3/795-804/.

23Freitas LC, Rodrigues GM, Araújo FC, Falcon EBS, Xavier NF, Lemos ELC, Pires CAA. Perfil dos hipertensos da Unidade de Saúde da Família Cidade Nova 8, município de Ananindeua-PA. Rev bras med fam comunidade. Florianópolis, 2012 [acesso em 2019 abr 29]; 7(22): 13-9. Disponível em:https://www.rbmfc.org.br/rbmfc/article/view/288/452.

24Pimenta AM. Epidemiologia da hipertensão arterial em uma comunidade rural do Vale do JequitinhonhaMG. [dissertação] Belo Horizonte: Escola de Enfermagem da Universidade Federal de Minais Gerais; 2005.

25Castro AP, Escatena MCM. Manifestação emocional de estresse do paciente hipertenso. Rev Latinoam Enferm. 2004 [acesso em 2019 abr 29]; 12(6):859-65. Disponível em: http://www.scielo.br/scielo.php?pid=S010411692004000600003\&script=sci_abstract\&tlng=pt.

26Sarno F, Claro RM, Levy RB, Bandonil DH, Monteiro CA. Estimativa de consumo de sódio pela população brasileira, 2008-2009. Rev Saúde Pública. 2013 [acesso em 2019 abr 29];47(3):571-578. Disponível em: http://www.scielo.br/pdf/rsp/v47n3/0034-8910-rsp-47-03-0571.pdf,08/11/18, 21:15.

27Magnanini MMF, Nogueira AR, Carvalho MS, Bloch KV. Monitorização ambulatorial da pressão arterial e risco cardiovascular em mulheres com hipertensão resistente. Arq Bras Cardiol. 2009[acesso em 2019 abr 29]; 92(6): 484-489. Disponível em: http://www.scielo.br/pdf/abc/v92n6/a12v92n6.pdf.

28Jesus ES, Augusto MAO, Gusm J, Júnior DM, Ortega K, Pierin AMG.Perfil de um grupo de hipertensos: aspectos biossociais, conhecimentos e adesão ao tratamento. Acta Paul Enferm 2008 [acesso em 2019 abr 29];21(1): 59-65. Disponível em: http://www.scielo.br/pdf/ape/v21n1/pt_08.pdf.

29Girotto E, Andrade SM, Cabrera MAS. Prevalência de Obesidade Abdominal em Hipertensos Cadastrados em uma Unidade de Saúde da Família. Arq Bras Cardiol. 2010[acesso em 2019 abr 29]. Disponível em: http://www.scielo. br/pdf/abc/v94n6/aop04610.

30Hasselmann MH, Faerstein E, Werneck GL, C, D, Lopes CS. Associação entre circunferência abdominal e hipertensão arterial em mulheres: Estudo Pró-Saúde. Cad. Saúde Pública.Rio de Janeiro; 2008[acesso $\begin{array}{lllll}\text { em } 2019 & \text { abr } & 29] & 24(5): 1187-1191 . & \text { Disponível }\end{array}$ http://bvsms.saude.gov.br/bvs/is_digital/is_0208/pdfs/IS28(2)048.pdf. 